\title{
Écoulement \\ dans les digues et barrages en terre \\ Modèles analogiques. Modèles physiques. \\ Que représente la notion de surface libre?
}

\author{
par G. Vachant
}

Maître de Recherches au C.N.R.S.

Institut de Mécanique de Grenoble

U.S.M.G.

Les modèles numériques et analogiques classiquement adoptés pour traiter les problèmes d'écoulement à surface libre dans des milieux poreux reposent sur l'hypothèse que tous les transferts ont lieu dans une zone saturée, de perméabilité constante, limitée par la surface libre. En cas d'écoulement transitoire (par exemple vidange rapide), on suppose de plus que tout le volume d'eau perdue par le sol provient de la variation de niveau de la «surface libre» (notion de porosité effective).

Le but de cette présentation est de discuter la signification physique de la notion de surface libre, afin de montrer qu'en régime transitoire les hypothèses généralement adoptées sont beaucoup trop simplifiées, ne représentent en rien l'évolution réelle des phénomènes, et conduisent aux résultats suivants :

- surévolution des volumes drainés;

- sous évaluation très forte de la durée du régime transitoire.

Ces résultats s'appuient sur deux séries d'essais effectués en similitude. Ils ont été obtenus lors de drainages d'une bande de sol de $3 \mathrm{~m}$ de long et $2 \mathrm{~m}$ de haut.

Dans un premier cas (modèle physique), on a réalisé une tranche de sol de $5 \mathrm{~cm}$ d'épaisseur ayant les dimensions décrites ci-dessus. Des essais de drainage ont été effectués sur cette tranche de sol, correspondant à des conditions de vidange rapide, et l'on a mesuré, par atténuation de rayon gamma et par tensiométrie, l'évolution des champs de teneur en eau, de pression et de potentiel dans toute la tranche de sol, ainsi que les variations de volume drainé.

Dans un second cas (modèle analogique), les mêmes séries d'essais ont été effectuées en similitude sur un modèle analogique de Hele-Shaw. Partant des mêmes profondeurs relatives de la nappe à surface libre, et imposant la même vidange, on a mesuré à partir de photographies rapides, l'évolution de la surface libre et des volumes drainés au cours du temps.

On trouvera (fig. 1) l'évolution relative des volumes écoulés obtenue avec le modèle physique et avec le modèle analogique (ramené en valeur réelle) lors d'un essai cor-

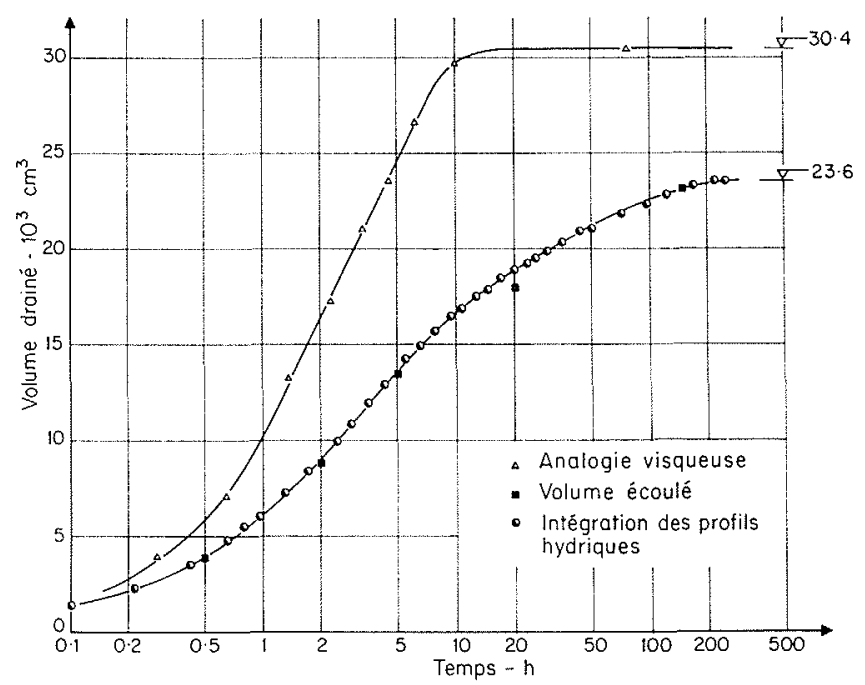

1/ Essals de rabattement. Comparaison entre les volumes d'eau écoulés par destockage du sol, et les volumes obtenus par analogie visqueuse. 

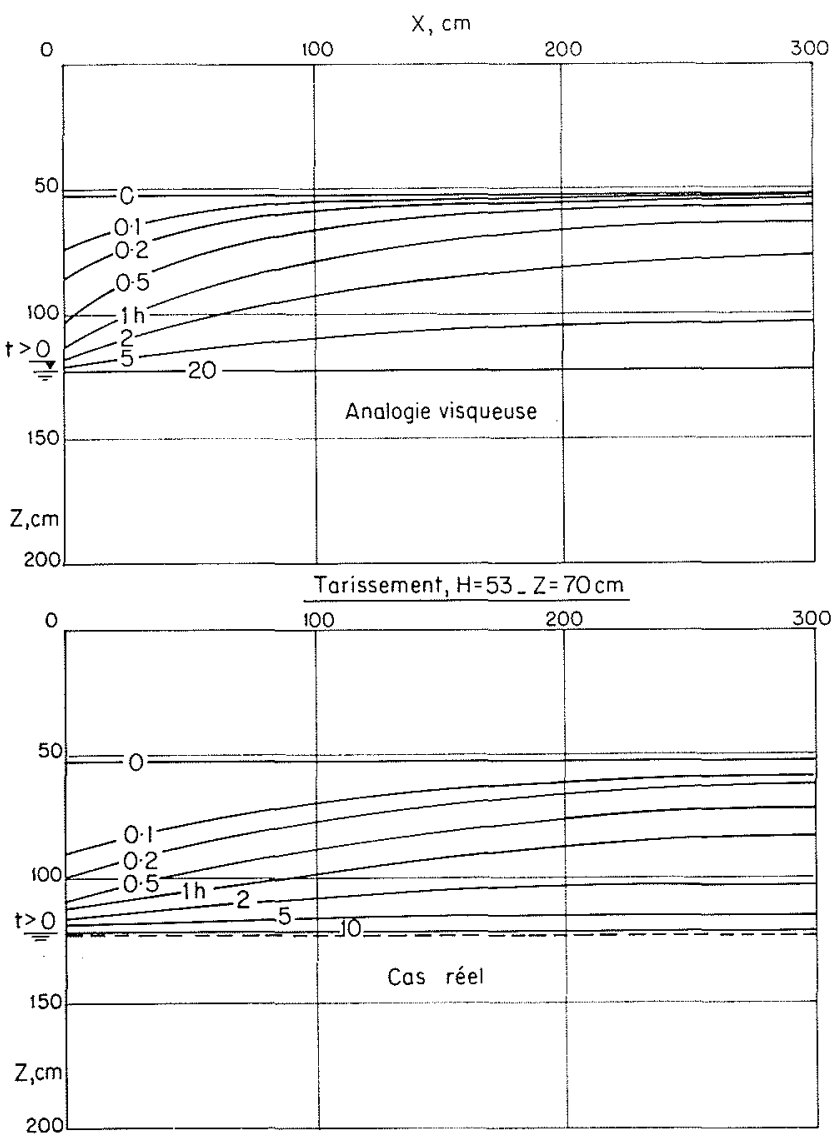

2/ Evolution de la surface libre, mesurée en analogie visqueuse et dans la tranche de sol, durant l'essai de tarissement.

respondant à un tarissement. Il est clair que les volumes obtenus par modèle analogique sont beaucoup plus forts que les volumes réellement écoulés (la différence représentant la quantité d'eau qui reste stockée dans le sol par capillarité), alors que la durée de l'écoulement est beaucoup plus courte $(5 \mathrm{~h}$ au lieu de $200 \mathrm{~h}$, cette différence étant due à l'utilisation d'un coefficient de perméabilité constant).

On a reporté (fig. 2) l'évolution relative des surfaces libres pendant le même essai. Il est paradoxal de trouver qu'à tout instant le rabattement de la «surface libre» lors de l'essai sur modèle réel est plus important que lors de l'essai analogique, alors que les volumes écoulés varient dans le sens inverse. Ce «paradoxe $»$ provient en fait de l'ambiguitté de la notion de «surface libre» qui n'a pas le même sens dans les deux cas :

- dans le modèle physique la surface libre est définie, comme le lieu des points où la pression de l'eau est atmosphérique, et elle est ainsi mesurée. C'est là son sens strict. Mais elle ne limite pas le domaine d'écoulement, il existe au-dessus de la surface libre une zone très importante : zone non saturée, qui contrôle d'autant plus la durée de l'écoulement que la perméabilité du sol diminue très fortement au fur et à mesure qu'il se désature. Noter ainsi qu'au bout de $10 \mathrm{~h}$ la surface libre est pratiquement en sa position d'équilibre, alors que plus d'un quart du volume total d'eau drainé va encore s'écouler entre 10 et $200 \mathrm{~h}$, sans variation de niveau de la surface libre, par simple drainage gravitaire de la zone non saturée. Du fait de l'existence de cette zone non saturée, notons enfin qu'il n'existe aucune relation directe entre variation de pression (surface libre) et variation de volume : une forte variation de pression (de 0 à $0,1 \mathrm{~h}$ ) peut très bien ne produire qu'une très faible variation de volume.

- dans le modèle analogique au contraire (et dans tout modèle mathématique classique) on suppose qu'il existe une relation directe entre variation de volume et variation de niveau de la surface libre, la première étant donnée par l'intégrale de la seconde multipliée par la porosité effective. On néglige de plus tout effet retardateur de la zone non saturée, ainsi que tout le stockage dû aux effets de rétentions capillaires.

Pour ces diverses raisons, on veillera à restreindre l'utilisation et la signification des résultats obtenus sur modèles analogiques et numériques basés sur l'intégration des équations de Boussinesq ou de Dupuit-Forchheimer, et l'on essayera plutôt d'utiliser des modèles numériques plus complets, basés sur des hypothèses de calcul plus réalistes.

On trouvera un exposé détaillé des méthodes expérimentales utilisées et des résultats obtenus en se reportant à l'article suivant:

G. Vachaud, M. Vauclin et D. KhanjI. - Etude expérimentale des transferts bidimensionnels dans la zone non saturée. Applications à l'étude du drainage d'une nappe à surface libre. La Houille Blanche, $\mathrm{n}^{\circ}$ 1, p. 65-73, 1973. 


\section{Discussion}

Présidents: MM. G. Kovacs et P. HABIB

M. le Président Habib remercie M. Vachaud de son exposé très suggestif et ouvre la discussion.

M. le Professeur THIRriot plaide pour le modèle Hele-Shaw Il pense qu'en le traitant comme un modèle analogique, il est possible de prendre en compte l'efiet des forces capillaires dans la zone non saturée.

En effet, dit-il, si l'on reprend celle de vos planches où l'on note l'évolution des volumes en fonction d'une part, de la porosité efficace ou effective, et d'autre part, des mesures de volume dans le modèle physique, on se rend compte qu'en jouant à la fois sur une échelle de temps et sur une échelle de hauteur, on devrait arriver à ajuster les résultats donnés par le modèle physique d'une part et le modèle Hele-Shaw d'autre part.

M. Vachaud n'est pas d'accord sur cette conclusion. Les courbes de variation de volume en fonction du temps sont, en effet, données en échelles semi-logarithmiques. Même si l'on ajuste la valeur du coefficient de porosité efficace pour avoir le même volume final, les échelles du temps sont encore très distordues.

D'autre part, poursuit M. le Professeur Trirrior, les modèles Hele-Shaw liennent compte des phénomènes de rétention. Pourquoi? Parce que, lorsqu'on prend un fluide relativement visqueux, il $y$ a un écoulement rampant le long de la glace et des auteurs russes, le Professeur Aravin et son élève, Mme Moscova, ont montré qu'ainsi on pouvait avoir une rétention non négligeable.

De mon côté, il y a trois ans, j'avais proposé de construire un modèle Hele-Shaw avec petits godets latéraux, permettant de représenter aussí bien les écoulements en milieu non saturé avec, en outre, la courbe d'hystérésis concernant le drainage de l'imbibition en prenant des ajustages dissymétriques qui étaient inspirés par les travaux de notre Maître, le Professeur Escande, sur les cheminées d’équilibre.

M. VACHaud répond en ces termes :

Un modele n'est valable que «s'il est en similitude». Un modèle Hele-Shaw est valable pour simuler les écoulements en milieu saluré car on montre physiquement qu'il existe une similitude de l'écoulement du fuide visqueux entre deux plaques parallèles avec l'écoulement d'un fluide visqueux (qui est l'eau) dans un milieu poreux (le sol) de perméabilité constante.

Dans la zone non saturée, le problème serait d'avoir une similitude entre la perméabilité qui varie très fortement en fonction de la teneur en eau et le fait, qu'en modèle Hele-Shaw, le fluide puisse ruisseler contre les parois. Je ne pense pas que cette similitude soit très correcte même si l'on munit le modèle d'ajutage ainsi que vous l'avez proposé.

Tout le problème est de pouvoir, avec un modèle analogique, réussir à simuler les relations très fortement non linéaires entre d'une part le degré de saturation et la conductivité hydraulique, d'autre part le degré de saturation et la pression capillairc. Ces deux relations fondamentales caractérisent, pour un sol donné, le comportement du fluide dans la zone non saturée pour des conditions initiales et aux limites données. Cette simulation analogique me semble si complexe que je lui préfère, de très loin, une simulation numérique.

M. Minetro (Instituto Superior Tecnico, Lisbonne) pose les deux questions suivantes :

$1^{\circ}$ Quelles sont les caractéristiques granalométriques du sol essayé ?

$2^{\circ}$ Quelle est la relation entre le volume accumulé dans la zone non saturée et le volume total drainé?

Nous utilisons un sable dont $100 \%$ des grains ont un diamètre inférieur à $1 \mathrm{~mm}, 50 \%$ inférieur à $0,2 \mathrm{~mm}$ et $0 \%$ au-dessous de $0,1 \mathrm{~mm}$. La frange capillaire s'étend sur une vingtaine de centimètres, ce qui est très faible sur une colonne d'essai de $2 \mathrm{~m}$ de hauteur répond $M$. VACHAUD.
M. le Professeur Boreli résume son opinion en ces termes:

Dans l'étude des écoulements transitoires des eaux souterraines à surface libre par les modèles Hele-Shaw ou n'importe quelle autre méthode se basant sur la notion de porosité effective, $\varepsilon$, existe le problème de préciser la valeur de $\varepsilon$. Dans une étude antérieure, nous avons démontré que $\varepsilon$ n'est pas une constante et que sa valeur dépend en premier lieu du rapport:

Vitesse du rabattement de la surface libre. conductivité

qui est en fonction directe avec le rapport de la vitesse du rabattement du niveau de l'eau externe à la conductivité. Dans le cas concret étudié, ce dernier rapport était pratiquement égal à l'infini, ce quí avait provoqué une variation importante de $\varepsilon$. L'auteur a donc soumis le modèle Hele-Shaw à des épreuves très difficiles, ce qui était tout à fait logique pour l'étude qu'il avait imaginée. Si le rabattement externe est plus lent, des conditions plus favorables existent pour l'application des modèles Hele-Shaw.

M. VAChaud répond à $M$. Boreli que des résultats semblables à ceux présentés lors de ce séminaire ont été obtenus lors d'essais de pompage, où le paramètre défni par M.. Borelr avait une valeur finie.

Avez-vous procédé à des essais sur la recharge des nappes, phénomène inverse de celui que vous avez étudié ? demande $M$. le Président Habib à M. Vachaud.

Il existe répond ce dernier un rapport D.G.R.S.T. sur ce sujet. Il est apparu qu'ici le modèle de Hele-Shaw est encore moins valable que dans le cas du drainage, puisque le modèle Hele-Shaw suppose que la recharge de la nappe est instantanée : on injecte all sommet du modèle et le fluide va instantanément à la nappe.

Dans la réalité, l'eau peut mettre plusieurs heures pour s'écouler depuis la surface du sol jusqu'à la nappe, mais la durée de ce transfert ne peut absolument pas être simulée par analogie visqueuse.

Tout en se défendant de vouloir prolonger la discussion avec M. VAChaUd, M. le Professeur THrrriot signale qu'il a pu étudier, sur modèle Hele-Shaw convenablement adapté, divers problèmes pratiques et, en particulier, ceux concernant la recharge d'une nappe soumise à des fluctuations périodiques; bien entendu, le procédé n'est pas applicable lorsque la présence d'air conduit localement à des écoulements biphasiques.

M. Sormall précise le point suivant :

Dans les digues en terre ou les sols argileux classiques, la lenteur des phénomènes de filtration ou de drainage est telle que la variation, avec le temps, de la teneur en eau au cours de certains phénomènes périodiques est faible: cette teneur en eau peut alors être considérée comme pratiquement constante. Dans de telles conditions, les hypothèses du modèle Hele-Shaw restent valables, peut-être plus que dans les cas correspondant à vos essais, où le sable choisi était très perméable et les modifications de sa perméabilité importantes.

En effet, observe M. VACHAUd, si les écoulements sont permanents ou quasi-permanents le problème est très différent mais, dans mes essais, je me suis placé dans un cas permettant l'étude des phénomènes transitoires.

Personne ne demandant à intervenir, M. le Président Habib clôt la discussion en remerciant tous ceux qui ont contribué à l'animer. Il donne ensuite la parole à $M$. BLANC pour l'exposé de sa communication. 